\title{
Satellite data analysis for identification of groundwater salinization effects on coastal forest for monitoring purposes
}

\author{
M. BARBARELLA ${ }^{1}$, M. DE GIGLIO ${ }^{1}$, L. PANCIROLI ${ }^{1}$ \& N. GREGGIO ${ }^{2}$ \\ 1 DICAM - Civic, Chemical, Environmental and Materials Engineering Deptartment, University of Bologna, \\ Via Risorgimento 2, I-40136 Bologna, Italy \\ maurizio.barbarella@,unibo.it \\ 2 CIRSA - Interdepartmental Research Centre for Environmental Sciences, University of Bologna, via St. Alberto 163 \\ I-48100, Ravenna, Italy
}

\begin{abstract}
In the phreatic aquifer below the San Vitale pinewood (Ravenna, Italy), natural and anthropogenic land subsidence, the low topography and the artificial drainage system have led to widespread saltwater intrusion. Since changes in the groundwater concentration induce variations in the vegetation properties, recognizable by different spectral bands, a comparison between satellite images, ASTER and Worldview-2, was made using the NDVI. The aim was to identify the portions of pinewood affected by salinization through a procedure that could reduce the expensive and time consuming ground monitoring campaigns. Moreover, the Worldview-2 high resolutions were used to investigate the Thermophilic Deciduous Forest (TDF) spectral behaviour without the influence of the allochthonous Pinus pinea species that is scattered throughout the pinewood. The NDVI, calculated with traditional bands, identified the same stressed areas using both satellite data. Instead, the new Red-Edge band of the Worldview-2 image allowed a greater correlation between NDVI and groundwater salinity.
\end{abstract}

Key words NDVI; groundwater salinity; coastal aquifer; ASTER, WorldView-2; stressed vegetation

\section{INTRODUCTION}

The goal of this study is to develop a procedure based on satellite remote sensing data that allows fast periodic monitoring of large areas that are exposed to groundwater salinization processes. Once the portions of pinewood affected by salt stress are identified it would be possible to focus only on the impacted areas ground monitoring campaigns, rescue operations and defensive works.

Several studies used the NDVI to analyse indirect effects of environmental changes (Aguilar et al. 2012; Barton 2012), including those due to processes of salinization (Zhang et al. 2011). Increased water salinity induces changes in chlorophyll concentration and therefore a photosynthesis slowdown (DeLaune et al. 1987). By measuring the relative difference between responses of chlorophyll and cellular structure in the red and near-infrared bands (Peñuelas, 1998), the NDVI analyses the greenness and productivity (Reed et al. 1994) of the plants.

In this work, the multispectral satellite data analysis is based on two images, Worldview-2 (WV-2) and ASTER, acquired in May 2011. In order to study the possible advantages of high spatial resolution compared to medium resolution, the results of both remotely sensed data elaborations were compared to verify if the same stressed areas were recognized (Pu and Landry, 2012; De Giglio et al., 2014). Subsequently, the higher spatial resolution and innovative spectral resolution of WV-2 (such as Red-Edge band) were used to perform an in-depth examination of the TDF spectral response (Wolf, 2010). Within the target vegetation, a high spatial variability of native species is present (Piccoli et al. 1991). Moreover, throughout the pinewood, the allochthonous Pinus pinea species is present but was excluded from the official vegetation maps.

\section{STUDY AREA}

The San Vitale pinewood is a narrow forest belt (Fig. 1(a)), $10 \mathrm{~km}$ long and $1.2 \mathrm{~km}$ wide (1200 ha). Despite the pinewood lying on palaeodunes, San Vitale has a very low topography, especially in the southern part where the average elevation is $0.3 \mathrm{~m}$ a.s.l. Moving northwards the elevation increases to $2 \mathrm{~m}$ a.m.s.l. The pinewood is surrounded by drained agricultural areas to the west, an industrial area in the south and the Piallassa Baiona lagoon, which is open to the sea, is the eastern boundary. Mollema et al. (2013) described a strong west-east salinity gradient (from 0.5 to $20 \mathrm{~g} / \mathrm{L}$ ) caused by the seepage between the saline surface water from the Piallassa and the groundwater of the pinewood. 


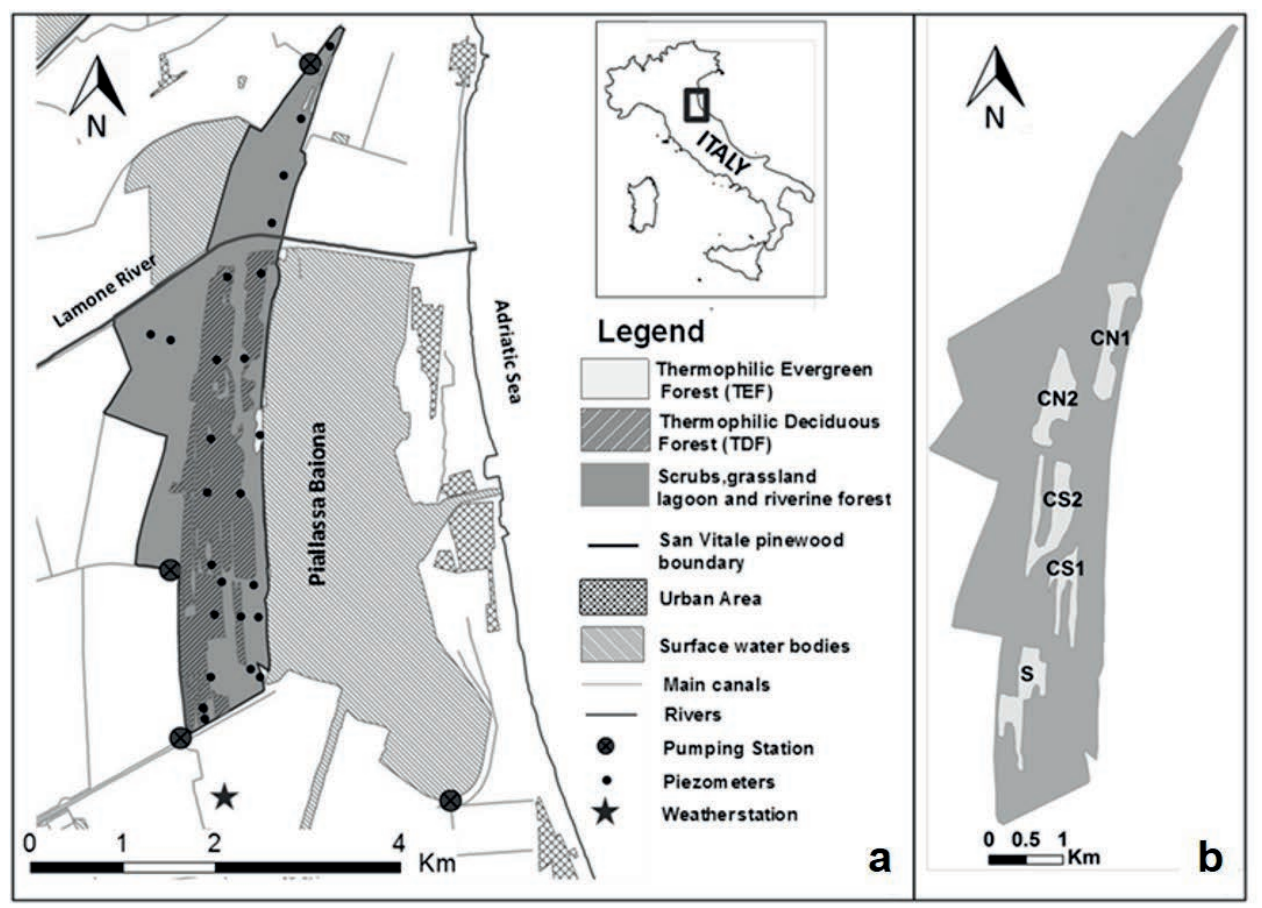

Fig. 1 San Vitale pinewood map with: (a) the vegetation types and the main hydrologic characteristics (brackish lagoon - Piallassa, rivers, main drainage channels), and (b) identification of the AOIs.

The forest is developed on dune ridges and slacks and the anthropogenic changes (subsidence, hydrologic management), have caused an articulated vegetation structure (Pirola 1974). In the official vegetation map the San Vitale woody cover is mainly constituted (55\%) by "Thermophilic Deciduous Forest" (TDF), that include Quercus robur, Quercus pubescens, Fraxinus ornu, Salix cinerea, Populus alba and Ulmus minor. The second order present (5\%) "Thermophilic Evergreen Forest" (TEF) is composed by the same species as above but Quercus ilex is the most common oak species. The remaining vegetation was grouped in this paper in "Shrubs, grassland, lagoonal and riverine forest". The Pinus pinea species is excluded from the classification because it was introduced by man since the 13th century and is not able to reproduce itself inside the natural area (Padula 1968; Piccoli et al. 1991). This species, is distributed inhomogeneously, especially along the morphological highs, and is stressed because it is outside of its original climax.

\section{METHODS}

In this study, the comparison between two satellite images with different spatial and spectral resolutions, ASTER (VNIR, 15-m and three bands) and WV-2 (2-m and eight bands), was made after the application of the procedure summarized in Fig. 2(a). The two datasets were acquired respectively on $05 / 18 / 2011$ and $05 / 29 / 2011$. In the first step, the NDVI was implemented using the same traditional bands (below tNDVI), Red $(0.630-0.690 \mu \mathrm{m})$ and NIR $(0.780-0.860 \mu \mathrm{m})$ for both images. While in the second step, the new WV-2 band Red-Edge (RE, 0.705-0.745 $\mu \mathrm{m}$ ) was implemented in the NDVI calculation (below nNDVI). The NDVI was calculated for the same five Areas of Interest (AOIs, Fig. 1(b)), selected inside the TDF class in both satellite data (CN1, CN2, CS1, CS2, S). For each AOI, the NDVI values were statistically evaluated. In every image, the threshold of 5\% for the AOI with higher average NDVI value were selected as criteria to compare the vegetation health status. Later, the percentages of pixels that fall below this limit were used to quantify the stressed vegetation in each other AOIs.

The validation of these results was made using a contemporaneous groundwater salinity monitoring and also the density of Pinus pinea was included in the final consideration.

In the third step the areas covered by Pinus pinea were separated from the rest of the TDF vegetation. The WV-2, the NEW TDF and PINE sub-AOIs were manually created within the main 
AOIs, using alternative combinations of bands (Digital Globe, 2013). With the RGB combination built with NIR1, RE, RED bands respectively, three kinds of coverage were identified based on different colour. The green and brown shades indicate coniferous trees (grey polygons in Fig. 2(b)), the yellow and orange shades identify deciduous forest (white polygons in Fig. 2(b)) and cultivated areas, bright yellow shows grassland and finally the grey shades correspond to bare soil and rock. Another colour combination was used to confirm the previous sub-AOIs. Considering NIR1, GREEN, BLUE bands it was possible to discriminate the coniferous trees (dark red, indicated by grey polygons in Fig. 2(c)), deciduous trees (bright red, indicated by white polygons in Fig. 2(c)) and grassland areas (red/orange).

However, while the pine trees are easily distinguishable with these colour combinations, the vegetation species that constitute the TDF class are not recognizable without an accurate field survey. Thus, considering only WV-2 data, the same statistical procedure presented in Fig. 2(a) (grey pathway) was applied on the sub-AOIs belonging to all five AOIs.

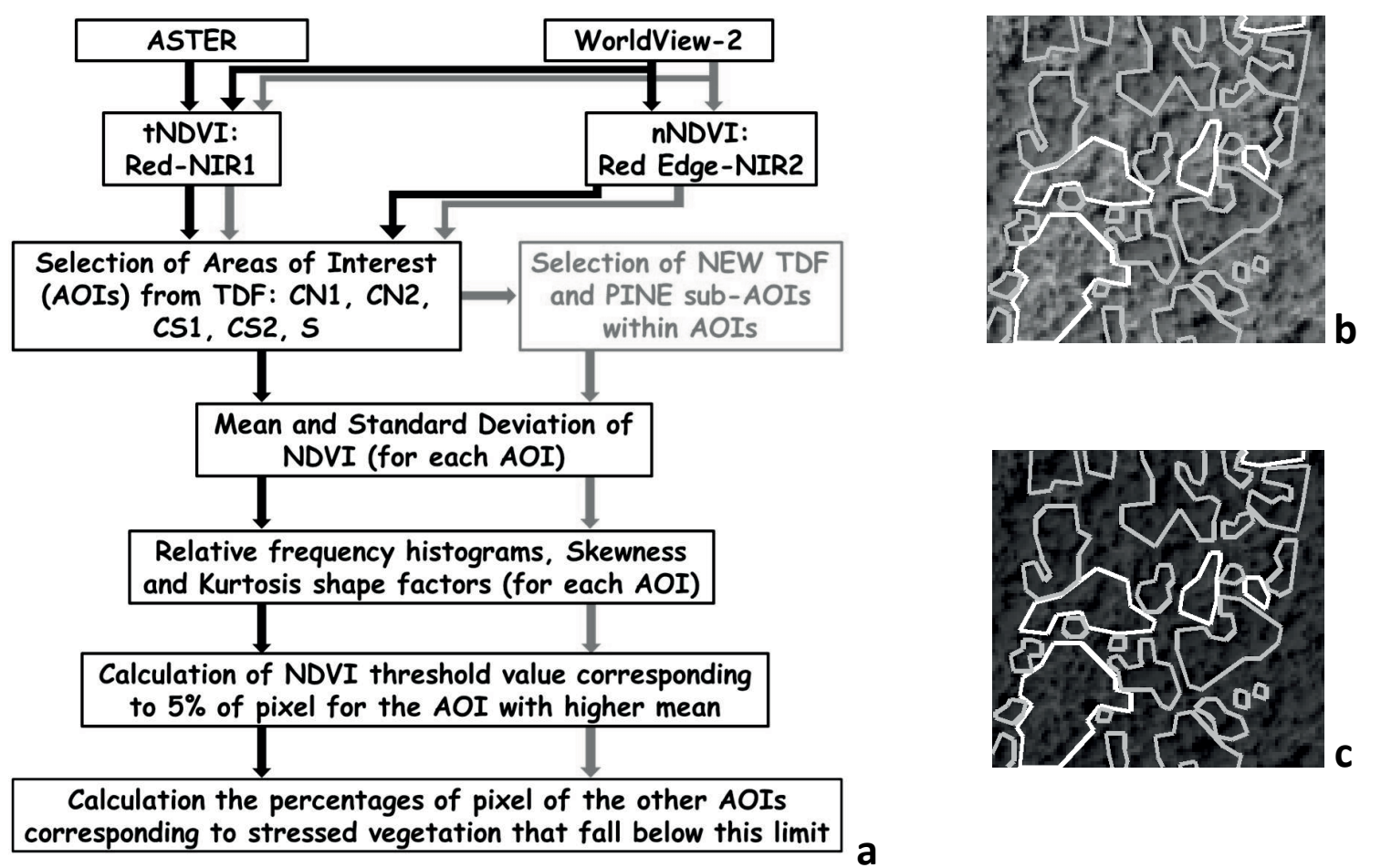

Fig. 2 (a) Flow chart of the procedure developed for AOIs (black pathway) and for sub-AOIs (grey pathway). (b) NEW TDF (white) and PINE (grey) Sub-AOIs examples for CN1 (highest pine tree density) NIR1-RE-RED band combination. (c) NEW TDF (white) and PINE (grey) Sub-AOIs examples for CN1 whit NIR1-GREEN-BLUE band combination.

\section{RESULTS AND DISCUSSION}

The average tNDVI values are coherent between ASTER and WV-2 satellite data (Fig. 3). The southern AOIs (CS2, CS1, S) were identified as less stressed areas compared to northern AOIs $(\mathrm{CN} 2, \mathrm{CN} 1)$. These results do not comply with the west-east salinity gradient. However, in the northern AOIs a higher pine tree density is present (Table 1) and it can be considered a negative factor for the overall health of the pinewood. The influence of the pines is more evident with the high spatial resolution. In fact, in the relative frequency histograms of CN1, CN2 and partly $\mathrm{S}$ generated from WV-2 elaboration data, NDVI value distributions have a bimodal trend, with the peak centred at lower NDVI values corresponding to pine species (Fig. 4(d)), as verified by further analysis. Instead, the nNDVI values computed using RE-NIR1 combination shows a different discrimination between AOIs that is perfectly consistent with the groundwater salinity values. 


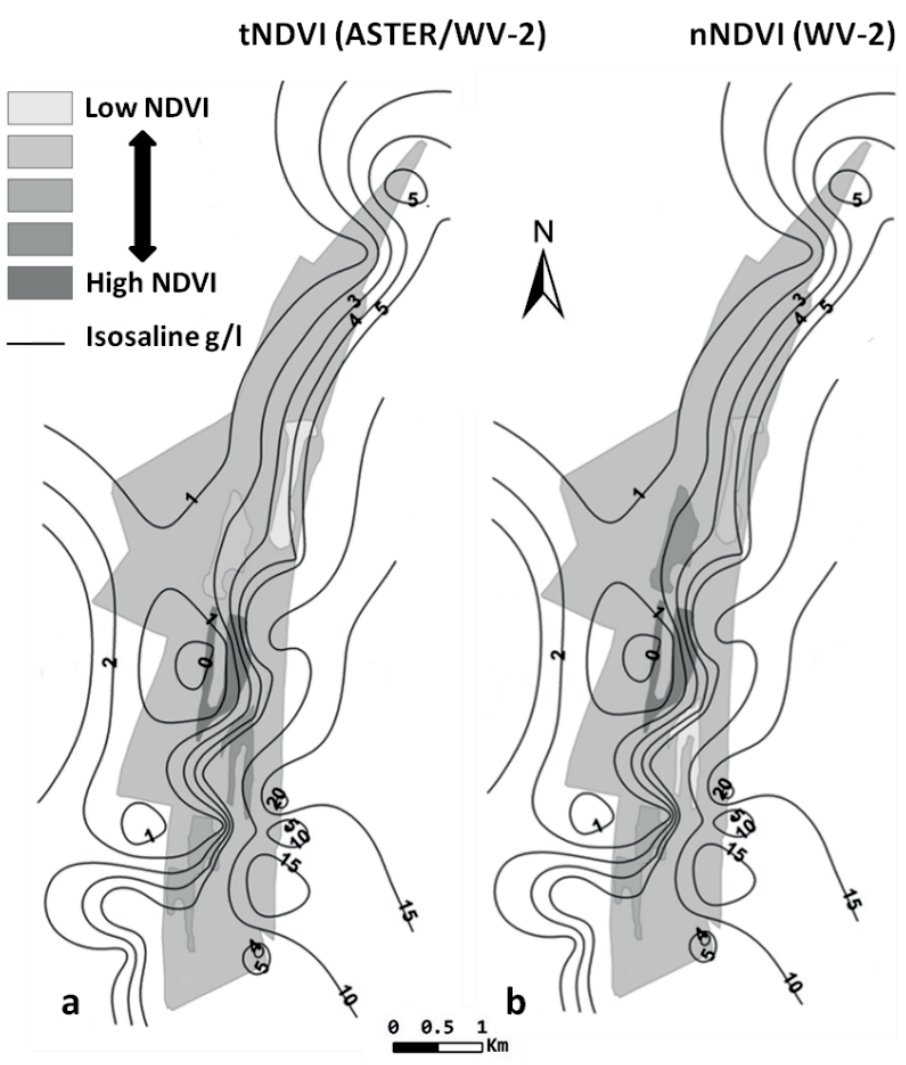

Fig. 3 Salinity maps for May 2011 and the ranking of the AOIs based on the average NDVI calculated with the traditional bands (a) and using the new RE band (b)

Table 1 Comparison between average tNDVI and nNDVI for each AOI and the corresponding average salinity and average pine tree density.

\begin{tabular}{lllll}
\hline AOI & Pine density No/ha & tNDVI (Aster/WV-2) & nNDVI (WV-2) & Salinity g/L \\
\hline 1 & CS2 (5.8) & CS2 $(0.841 / 0.850)$ & CS2 $(0.313)$ & CS2 (1.77) \\
2 & CS1 (7.6) & CS1 $(0.823 / 0.837)$ & CN2 $(0.304)$ & CN2 (2.19) \\
3 & S $(14.1)$ & S $(0.821 / 0.829)$ & S $(0.299)$ & S $(3.42)$ \\
4 & CN2 $(19.8)$ & CN2 $(0.813 / 0.826)$ & CN1 $(0.0 .298)$ & CN1(3.90) \\
5 & CN1 $(25.2)$ & CN1 $(0.799 / 0.805)$ & CS1 $(0.297)$ & CS1 (7.12) \\
\hline
\end{tabular}

After the application of the whole procedure for all calculated NDVI, the ranking of AOIs was done in terms of salinity, pine tree density and both tNDVI and nNDVI (Table 1).

From the sub-AOIs statistical analysis, the average tNDVI values of the NEW TDF sub-areas were higher than those of PINE sub-AOIs, as confirmed by their relative frequency histograms (examples in Fig. 4(b), 4(c), 4(e) and 4(f)). Considering the different species of vegetation and foliage, the lower tNDVI average values for the PINE sub-AOIs prove that these allochthonous plants are stressed regardless of their morphological position inside the pinewood and of the salinity conditions. The comparison between NEW TDF average tNDVI and the average groundwater salinity values confirms the west-east groundwater salinity gradient (Table 2). The western NEW TDF sub-AOIs (CS2, S and CN2) show higher values than eastern NEW TDF SubAOIs (CS1 and CN1). Thus, the use of the Sub-AOIs allows to overcome the previous mismatch between the areas with higher groundwater salinity but contemporary high NDVI values.

Also using the RE band, the average nNDVI of NEW TDF sub-AOIs was higher than those of PINE sub-AOIs. Consequently, the frequency histograms of the NEW TDF sub-AOIs reported higher NDVI with respect to the PINE sub-AOIs. 


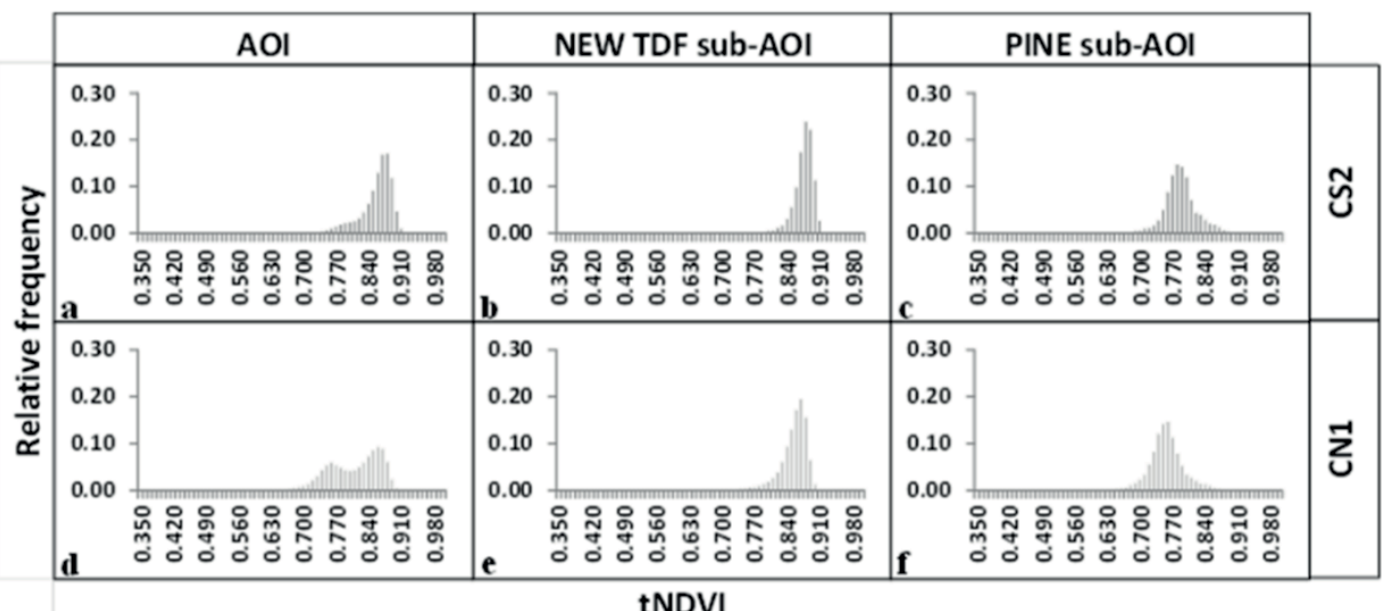

Fig. 4 Examples of tNDVI frequency histograms for CS2 and CN1 AOIs. For the entire AOIs (a) and (d), for NEW TDF (b) and (e) and for the PINE portion (c) and (f).

Table 2 Comparison between average tNDVI and nNDVI values for the NEW TDF sub-AOIs and the corresponding average salinity.

\begin{tabular}{llll}
\hline AOI & Salinity g/L & tNDVI & nNDVI \\
& & NEW TDF sub-AOIs & NEW TDF sub-AOIs \\
\hline 1 & CS2 $(1.77)$ & CS2 $(0.871)$ & S $(0.331)$ \\
2 & CN2 $(2.19)$ & S $(0.871)$ & CN2 $(0.330)$ \\
3 & S $(3.42)$ & CN2 $(0.870)$ & CS2 $(0.328)$ \\
4 & CN1(3.90) & CS1 $(0.866)$ & CN1 $(0.320)$ \\
5 & CS1 $(7.12)$ & CN1 $(0.850)$ & CS1 $(0.313)$ \\
\hline
\end{tabular}

Considering only the average nNDVI, the NEW TDF sub-AOI division confirms the westeast salinity gradient, but does not perfectly correspond to AOIs classifications based on groundwater salinity. The causes are the complex distribution of plant species inside TDF and the RE band's greater sensitivity to the variability and the health status of the vegetation. This behaviour is confirmed by the near-normal distribution of the relative frequency histograms for the TDF Sub-AOIs.

With the creation of NEW TDF sub-AOIs, the saltwater intrusion was accounted as the main stress factor due to exclusion of pine plants from the analysis. Consequently, the linear correlation between average salinity values and average NDVI values of NEW TDF sub-AOIs increases in the case of nNDVI (Fig. 5). Both linear regression lines confirm the negative correlation between salinity and NDVI.
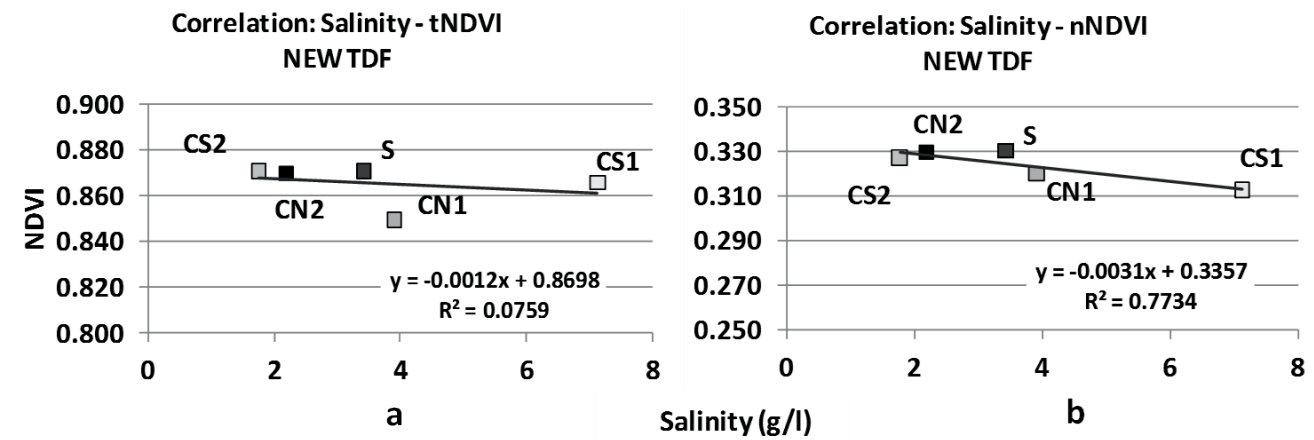

Fig. 5 Linear correlation between average salinity values and average tNDVI (a) and nNDVI (b) values of NEW TDF sub-AOIs. 
For tNDVI, the 5th percentile parameter of NEW TDF sub-AOIs does not supply useful information, while the second order moments, skewness and kurtosis, confirm a non-Gaussian distribution. tNDVI is less sensitive to the variety of species within TDF vegetation, but is more influenced by pine plants than nNDVI. Also for the nNDVI, the statistical analysis of NEW TDF sub-AOIs does not supply new useful information about AOIs conditions because of the Gaussian distribution of the relative frequency histograms for each of the Sub-AOIs. The higher sensitivity to the complex distribution of plant species inside NEW TDF areas for the RE band is confirmed.

\section{CONCLUSION}

The discrimination of the AOIs using the tNDVI is identical for ASTER and Worldview-2 data. Based on mean tNDVI value, the northern AOIs (CN2, CN1) were identified as more stressed areas compared to southern AOIs (CS2, CS1, S), in contrast to the west-east groundwater salinity gradient. However, the $\mathrm{CN} 2$ and $\mathrm{CN} 1$ AOIs are also the areas with the highest pine trees densities and their lower tNDVI values calculated on the PINE sub-AOIs confirm that the pine trees are a negative factor for the health of the overall pine forest. The higher sensitivity to the pine density of tNDVI can be deduced from the NEW TDF sub-AOIs values that, once deprived of Pinus pinea trees, confirm the west-east groundwater salinity gradient. The nNDVI values show different discriminations between AOIs that are perfectly consistent with the groundwater salinity values. Therefore, this nNDVI is less influenced by pine plant density than tNDVI. However, in terms of sub-AOIs, nNDVI is more sensitive to distribution of the species within TDF vegetation that is not uniformly distributed in the several sub-areas. This aspect is probably related to the rapid growth characterizing the vegetation spectral signature within the RE range. The problem is less evident at the AOIs scale because their compositions are globally more similar to each other. This study suggests concentrating the ground monitoring for salinity in the far eastern part of the pine forest. However, it also highlights that it is not possible to overlook other sources of stress present in the area.

\section{REFERENCES}

Aguilar, C., et al. (2012) NDVI as an indicator for changes in water availability to woody vegetation. Ecological Indicators 23, 290-300.

Barton, C.W.M. (2012) Advances in remote sensing of plant stress. Plant and Soil 354, 41-44.

De Giglio. M., et al. (2014) ASTER and WorldView - 2 satellite data applications for recognition of salt water intrusion on forest vegetation. 23rd Salt Water Intrusion Meeting, June 16-20, 2014, Husum, Germany. Proceedings 75-78.

DeLaune, R.D., Pezeshki, S.R. and Patrick Jr., W.A. (1987) Response of coastal plants to increase in submergence and salinity. Journal of Coastal Research 3(4), 535-546.

Digital Globe (2013) WorldView-2 Band Combinations. C-AGG (Coalition on Agricultural Greenhouse Gases) Meeting Summary, Detroit, Michigan-USA.

Mollema, P.N., et al. (2013) Hydrochemical and physical processes influencing salinization and freshening in Mediterranean low-lying coastal environments. Applied Geochemistry 34, 207-211.

Padula, M. (1968) Ricerche sulle condizioni ecologiche dei boschi di S. Vitale e di Classe (Ravenna). Annali dell'Accademia Italiana delle Scienze Forestali 17, 173-244.

Peñuelas, J. (1998) Visible and near-infrared reflectance techniques for diagnosing plant physiological status. Trends in Plant Science 3, 151-156.

Piccoli, F., Gerdol, R. and Ferrari, C. (1991) Vegetation Map of St. Vitale pinewood (Northern Adriatic coast, Italy). Phytocoenosis, 337-342.

Pirola, A. (1974) La vegetazione della Pineta di San Vitale. Influenza di insediamenti industriali sul circostante ambiente naturale. Studio sulla Pineta di San Vitale di Ravenna (pp 76-88). Ed. Compositori. Bologna, Italy: Ed. Compositori.

$\mathrm{Pu}, \mathrm{R}$. and Landry, S. (2012) A comparative analysis of high spatial resolution IKONOS and WorldView-2 imagery for mapping urban tree species. Remote Sensing of Environment 124, 516-533.

Reed, B., et al. (1994) Measuring phenological variability from satellite imagery. Journal of Vegetation Science 5, 703-714.

Wolf, A. (2010) Using WorldView2 VisNIR MSI imagery to support land mapping and feature extraction using normalized difference index ratios. DGI 8BandResearch Challenge, 1-13.

Zhang, T., et al. (2011) Using hyperspectral vegetation indices as a proxy to monitor soil salinity. Ecological Indicators 11, $1552-1562$ 